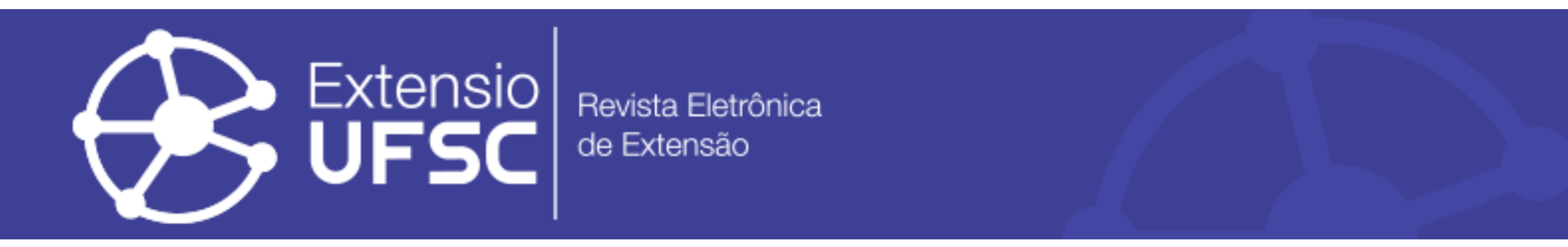

\title{
IMPACTO DE UM PROJETO DE EXTENSÃO SOBRE A FORMAÇÃO DISCENTE PARA ATUAÇÃO EM AMBIENTE HOSPITALAR
}

\author{
Gabriela dos Passos Rodrigues \\ Universidade Federal de Santa Catarina \\ rodrigues.gp@hotmail.com \\ Giovanna Steffenello Durigon \\ Universidade Federal de Santa Catarina \\ giovanna.steff@gmail.com \\ Mariah Luz Lisboa \\ Universidade Federal de Santa Catarina \\ mariah.lisboa@gmail.com \\ Augusto Bodanezi \\ Universidade Federal de Santa Catarina \\ abodanezi@hotmail.com
}

Beatriz Álvares Cabral de Barros

Universidade Federal de Santa Catarina beatriz.barros@gmail.com

Thais Mageste Duque

Universidade Federal de Santa Catarina thaismadu@hotmail.com

Etiene de Andrade Munhoz Universidade Federal de Santa Catarina timunhoz@gmail.com

Inês Beatriz da Silva Rath Universidade Federal de Santa Catarina ibrath@gmail.com

Alessandra Rodrigues de Camargo Universidade Federal de Santa Catarina alessandra.camargo@ufsc.br

\section{Resumo}

O papel da Universidade é baseado em um processo interdisciplinar, educativo, cultural, científico e político, promovendo uma interação transformando não apenas a Universidade, mas também setores sociais com os quais interage. Objetivo: (1) descrever o trabalho realizado e a experiência adquirida dentro de um projeto de extensão universitária da área de saúde intitulado "Ambulatório de Odontologia Oncológica"; (2) expor a importância do atendimento odontológico promovido pelo projeto no Hospital Universitário da Universidade Federal de Santa Catarina (HU/UFSC). Resultados: ficou evidenciado que a extensão propicia o exercício da atuação multiprofissional e interdisciplinar, dando uma visão holística do paciente, muito além de suas necessidades odontológicas. Conclusão: os atendimentos odontológicos promovidos pelo projeto Ambulatório de Odontologia Oncológica - Onco-hematologia são essenciais para a atenção integral do paciente oncológico, além da formação técnico-científica adicional que alunos de graduação extensionistas do projeto recebem. Propõe-se que estas atividades práticas sejam incluídas na matriz curricular do curso.

Palavras-chave: Unidade Hospitalar de Odontologia. Oncologia. Manifestações Bucais. Educação em Odontologia.

\section{IMPACT OF AN EXTENSION PROJECT ON THE DISCIPLE TRAINING FOR ACTION IN A HOSPITAL ENVIRONMENT}

\begin{abstract}
The University's role is based on an interdisciplinary, educational, cultural, scientific and political process, promoting an interaction transforming not only the University, but also social sectors in which it interacts. Objective: (1) to describe the work accomplished and the experience acquired within a project of university extension of the health area entitled "Ambulatory of Oncological Dentistry"; (2) to explain the importance of dental care promoted by the project at the University Hospital of the Federal University of Santa Catarina (HU/UFSC). Results: it was evidenced that the extension provides the exercise of multiprofessional and interdisciplinary action, giving a holistic view of the patient, far beyond their dental needs. Conclusion: Dental care promoted by the Ambulatory Oncology: Onco-hematology is essential for the comprehensive care of the cancer patient, as well as the additional technical and scientific training undergraduate project extension students receive. It is proposed that these practical activities be included in the curricular matrix of the course.
\end{abstract}

Keywords: Dental Service Hospital. Oncology. Oral Manifestations. Dental Education. 


\title{
IMPACTO DE UN PROYECTO DE EXTENSIÓN SOBRE LA FORMACIÓN DISCIENTE PARA LA ACTUACIÓN EN AMBIENTE HOSPITALARIOL
}

\begin{abstract}
Resumen
El papel de la Universidad se basa en un proceso interdisciplinario, educativo, cultural, científico y político, promoviendo una interacción transformando no sólo la Universidad, sino también sectores sociales con los que interactúa. Objetivo: (1) describir el trabajo y la experiencia dentro de un proyecto de extensión universitaria de la salud titulado "Oncología Clínica Dental"; (2) exponer la importancia de la atención odontológica promovido por el proyecto en el Hospital Universitario de la Universidad Federal de Santa Catarina (HU / UFSC). Resultados: se evidenció que la extensión propicia el ejercicio de la actuación multiprofesional e interdisciplinaria, dando una visión holística del paciente, mucho más allá de sus necesidades odontológicas. Conclusión: las atenciones odontológicas promovidas por el proyecto Ambulatorio de Odontología Oncológica - Onco-hematología son esenciales para la atención integral del paciente oncológico, además de la formación técnico-científica adicional que alumnos de graduación extensionistas del proyecto reciben. Se propone que estas actividades prácticas se incluyan en la matriz curricular del curso.

Palavras clave: Unidad Hospitalaria de Odontología. Oncología. Manifestaciones Bucales. Educación en Odontología.
\end{abstract}


Impacto de um projeto de extensão sobre a formação discente para atuação em ambiente hospitalar

\section{INTRODUÇÃO}

A Odontologia Hospitalar $(\mathrm{OH})$ compreende os cuidados que os cirurgiões dentistas têm com pacientes presentes nos ambientes hospitalares. Esses cuidados exigem intervenções de equipes multidisciplinares, com atendimentos de alta complexidade e com o objetivo de melhorar o desempenho na assistência ao paciente (CAMARGO, 2005).

No Brasil, a OH foi legitimada em 2004 juntamente com a Associação Brasileira de Odontologia Hospitalar (ABRAOH), porém, somente em 2008, foi apresentada à Câmara dos Deputados do Rio de Janeiro o Projeto de Lei no 2776/2008 que estabelece a obrigatoriedade da presença de profissionais de Odontologia nas equipes multiprofissionais das Unidades de Terapias Intensivas (UTIs) (BRASIL, 2013).

Em outubro de 2014, a Assembleia Nacional de Especialidades Odontológicas (ANEO), reconheceu o exercício da $\mathrm{OH}$ pelo cirurgião-dentista (CD). De acordo com a Resolução 162 do Conselho Federal de Odontologia (CFO) de 03 de novembro de 2015, para obter seu registro no CFO e inscrição no Conselho Regional de Odontologia (CRO), o profissional deve realizar o curso de $\mathrm{OH}$ com um mínimo de 350 horas, sendo 30\% de horas práticas e 70\% de aulas teóricas e, ao final, ser aprovado na avaliação teórica e prática. Para dentistas que tenham atuado na área por no mínimo 05 anos, nos últimos 10 anos, tiveram, ainda, a possibilidade de requerer a habilitação mediante comprovação documental. Para tanto, exigiu-se a apresentação do contrato de trabalho ou declaração do CD com a instituição ou uma declaração do representante legal ou membro do corpo clínico do hospital. Os profissionais que não conseguiram provar, por meio de tais documentos sua inserção no ambiente hospitalar, podem realizar uma prova escrita além da análise de currículo (BRASIL, 2015). A partir da obtenção do referido registro, o cirurgiãodentista legalmente encontra-se apto a trabalhar no contexto hospitalar, sendo responsável por fomentar a sistematização de serviços (BRASIL, 2017a).

Os campos de atuação do CD nos serviços odontológicos hospitalares incluem: diagnóstico de lesões bucais e auxílio no tratamento de manifestações bucais oriundas de doenças sistêmicas; diagnóstico e tratamento das condições bucais que possam acarretar complicações infecciosas, hemorrágicas, neurológicas ou cardiovasculares, seja em função das condições locais e sistêmicas, seja em decorrência do tratamento ao qual o paciente está submetido; diagnóstico e tratamento das condições bucais que possam colaborar para a manutenção ou piora de desordens sistêmicas graves; atuação prévia a terapias que possam acarretar complicações orofaciais ou complicações sistêmicas futuras; atendimento a pacientes internados que apresentem dor e/ou infecção de origem odontológica; e atendimento de quaisquer condições que justifiquem 
Impacto de um projeto de extensão sobre a formação discente para atuação em ambiente hospitalar

intervenção em ambiente hospitalar, em função do risco de complicações infecciosas ou hemorrágicas tanto em nível local quanto sistêmico (SÃO PAULO, 2012).

A Unidade de Onco-hematologia do Hospital Universitário Professor Polydoro Ernani de São Thiago (HU/UFSC), em Florianópolis, Santa Catarina, trata das doenças malignas do sangue e sistema linfático. Fazem parte desse grupo os linfomas, leucemias, doenças mieloproliferativas (policitemia vera, trombocitopenia e mielofibrose), mielodisplasias e o mieloma múltiplo. Estas doenças exigem tratamento específico e uma equipe multidisciplinar, que reúna médicos, enfermeiros, fisioterapeutas, dentistas, nutricionistas, psicólogos, profissionais de medicina integrativa e complementar, além de assistentes sociais (SANTOS et al., 2016), visto que uma abordagem multidisciplinar é essencial para o tratamento de pacientes submetidos à terapia antineoplásica. Dessa forma cada especialidade, de acordo com seu nível de competência, contribui na solução de problemas no atendimento global do paciente com câncer (JOSHI, 2010).

A terapia antineoplásica, em especial a que trata das doenças onco-hematológicas, é complexa e consta de quimioterapia, radioterapia e o transplante de células tronco hematopoiéticas. Estes tratamentos são citotóxicos e possuem efeitos adversos em tecidos normais, os quais podem dificultar a continuidade da terapêutica, fato complicador para a expectativa da sobrevida do paciente. A quimioterapia pode afetar o organismo de forma local e/ou sistêmica, visto a gama de complicações orais, agudas e tardias, associadas às medicações administradas. Dentre elas, podemos citar mucosite, xerostomia, disgeusia, infecções fúngicas, bacterianas e virais, trismo e neurotoxicidade. Além disso, em pacientes pediátricos pode ocorrer o comprometimento da formação óssea, muscular e dentária (SONIS et al., 1996).

O manejo das complicações bucais resultantes da terapia antineoplásica, exige do CD atuações preventivas, curativas e paliativas; contudo, a falta de capacitação para atuação na alta complexidade, faz com que muitos alunos de graduação não se sintam confortáveis, ou poucos capacitados, para o trabalho em equipe multiprofissional indissociável no contexto hospitalar (PIVETTA et al., 2010).

Ao longo do Curso de Graduação em Odontologia da UFSC, o treinamento sobre o cuidado do paciente oncológico em regime de internação hospitalar é ofertado em poucas disciplinas teóricas. Mesmo assim acreditamos que a falta de atividades práticas complementares, não permite que o aluno de graduação atinja formação e qualificação suficientes para o atendimento odontológico deste público (UFSC, 2017).

Com a intenção de suprir essa lacuna, no ano de 2014, um grupo de professores, da Universidade Federal de Santa Catarina (UFSC), criou um projeto de extensão com oferta de campo de estágio para alunos do último ano do Curso de Graduação em Odontologia. Em linhas 
Impacto de um projeto de extensão sobre a formação discente para atuação em ambiente hospitalar

gerais, o projeto oferece ao aluno a possibilidade da vivência em um ambiente hospitalar, partilhando experiências com equipes multiprofissionais e com o próprio paciente.

Desta forma o presente trabalho teve como objetivos: 1) descrever o trabalho realizado e a experiência adquirida dentro de um projeto de extensão universitária da área de saúde intitulado "Ambulatório de Odontologia Oncológica: Onco-hematologia", criado no primeiro semestre de 2014;2) expor a importância do atendimento odontológico promovido pelo referido projeto no Núcleo de Odontologia Hospitalar e na Clínica Médica II do Hospital Universitário da Universidade Federal de Santa Catarina (HU/UFSC).

\section{MATERIAIS E MÉTODOS}

Trata-se de um trabalho observacional e de pesquisa nos arquivos do Núcleo de Odontologia Hospitalar do HU. Foi realizada busca nas bases de dados PubMed e Scielo com os descritores "Odontologia Hospitalar", "Oncohematologia" e "Odontologia em alta complexidade". Também foi efetuada busca no site da UFSC para estudo das diretrizes curriculares, no site do Conselho Federal de Odontologia para busca de normativas, além da base de dados gerada pelo projeto de extensão “Ambulatório de Odontologia Oncológica: Onco-bematologia”.

O referido Projeto se desenvolve no Núcleo de Odontologia Hospitalar e na Clínica Médica II do Hospital Universitário Professor Polydoro Ernane de São Thiago, da Universidade Federal de Santa Catarina (HU/UFSC).

Fisicamente, as instalações do Núcleo de Odontologia Hospitalar, estão localizadas no $3^{\circ}$ andar do Hospital Universitário Professor Polydoro Ernani de São Thiago. A estrutura conta com 1 recepção, 3 consultórios odontológicos (sendo 1 sala cirúrgica), 3 salas de entrevista, 1 consultório para terapias com laser de baixa potência, 1 expurgo, 1 sala de esterilização e 1 cozinha independente. Para desempenhar as atividades previstas no "Ambulatório de Odontologia Oncológica: Onco-hematologia" trabalham neste espaço, semanalmente, um cirurgião-dentista staff (preceptor), quatro residentes do Programa de Residência Integrada Multiprofissional em Saúde Alta Complexidade (dois residentes do primeiro ano e dois residentes do segundo ano), dois graduandos em Odontologia bolsistas do projeto pelo Edital PROBOLSAS, um graduando extensionista voluntário da $3^{\text {a }}$. Fase do Curso de Graduação em Odontologia para apoio do serviço, dez graduandos em Odontologia extensionistas voluntários que atuam na assistência ao paciente. Ademais, trabalham neste ambiente, seis professores do Departamento de Odontologia, que contemplam as especialidades de Estomatologia, Odontologia para Pacientes Especiais, Dentística, Odontopediatria e Endodontia, os quais atuam como tutores. Vale salientar que a 
Impacto de um projeto de extensão sobre a formação discente para atuação em ambiente hospitalar

descrição de alunos extensionistas supracitados considera todos aqueles que estão envolvidos diretamente neste projeto, uma vez que o Núcleo de Odontologia Hospitalar possui outros projetos em andamento e conta com outros voluntários.

\section{RESULTADOS E ANÁLISES}

As atividades clínicas relacionadas ao projeto de extensão "Ambulatório de Odontologia Oncológica: Onco-hematologia” ocorrem semanalmente, às terças-feiras, das 8:00hs - 12:00hs. Alunos extensionistas, bolsistas e voluntários participantes do projeto, são divididos em duas turmas, com escalas semanais rotativas (alternadas) para as seguintes atividades:

- Grupo de atendimento I: Visita ao leito de pacientes internados em Enfermaria no HU/UFSC, nas Clínicas Médica I (número de leitos flutuantes de acordo com a demanda) e II (9 leitos totais) pela equipe de Onco-hematologia. Nas visitas aos leitos dos pacientes internados, são efetuados diagnósticos, tratamentos e acompanhamento das lesões ou sintomas bucais, principalmente aqueles relacionados com os efeitos colaterais do tratamento antineoplásico. Nesse momento são realizadas discussões com a equipe multiprofissional que acompanha os internos, para tomada de decisões clínicas.

- Grupo de atendimento II: Atendimento ambulatorial no Setor de Odontologia Hospitalar do mesmo hospital, sob orientação de seis professores/tutores e um preceptor. Nos atendimentos ambulatoriais, são realizados procedimentos que objetivam eliminar focos de infecção, além de profilaxia e adequação bucal, tratamentos de canal, restaurações e pequenas cirurgias. Também são realizadas radiografias e exames complementares com a finalidade de diagnóstico $\mathrm{O}$ objetivo desse atendimento é preparar o paciente para as diferentes etapas da terapia antineoplásica, proporcionando melhores condições de saúde bucal. Pacientes com diagnósticos recentes de doenças onco-hematológicas são primariamente avaliados pela equipe médica e, após, são direcionados para avaliação odontológica.

Para ampliar os conhecimentos e formação dos participantes, quinzenalmente são realizados estudos de casos e discussões de artigos científicos, relacionados as doenças e particularidades destes pacientes, a rotina do serviço, a complexidade dos procedimentos e aos diversos tipos de tratamentos médicos aos quais estão sujeitos e, suas repercussões e importância na saúde bucal.

O Conselho Regional de Odontologia de Santa Catarina (CRO/SC) reconhece, até o momento, oito Cirurgiões Dentistas habilitados para o exercício da $\mathrm{OH}$. Dentre estes, três atuam no município de Florianópolis (dois desses estão em atividade no Hospital Universitário 
Impacto de um projeto de extensão sobre a formação discente para atuação em ambiente hospitalar

Professor Polydoro Ennani de São Thiago), três em Blumenau, um em Pomerode (cirurgiãodentista bucomaxilofacial do Hospital Santa Isabel) e um em Itajaí (BRASIL, 2017b). Vale a pena salientar ainda, que os profissionais habilitados em exercício no HU/UFSC, são professores do Curso de Odontologia que desenvolvem as atividades de extensão no Núcleo de Odontologia Hospitalar desse hospital.

Talvez o reconhecimento recente da área de habilitação pelo CFO seja o maior responsável pelo número escasso de profissionais em exercício regulamentados no $\mathrm{CRO} / \mathrm{SC}$, somado ao fato da falta de cursos em nossa região. Nesse sentido, o campo de estágio criado pelo projeto de extensão “Ambulatório de Odontologia Oncológica: Onco-hematologia” possibilita para os graduandos e extensionistas uma vivência de vanguarda na área de Odontologia Hospitalar.

O Curso de Graduação em Odontologia tem como objetivo promover um treinamento técnico científico do estudante com vistas à formação de um cirurgião-dentista clínico generalista preparado para o atendimento ambulatorial de pacientes em relativa condição de saúde (AMANTE, 2011). A proposta pedagógica do Curso de Graduação em Odontologia está fundamentada nas Diretrizes Curriculares e busca a formação do estudante através de um processo articulado de ensino, pesquisa e extensão. O objetivo dessa tríade é promover aprimoramento contínuo das ações e estimular propostas inovadoras de interação comunitária, bem como ampliar e melhorar as ações de interação com os setores organizados da sociedade. $\mathrm{O}$ currículo apresenta um eixo temático central: uma Odontologia que promove saúde, voltada para realidade da população brasileira. A vertente extensionista proposta pelo programa propicia a vivência de situações externas ao ambiente da universidade, a partir da interação com a população assistida, pertencente a diferentes realidades sociais, econômicas, culturais e de saúde (UFSC, 2015).

Além da matriz curricular obrigatória e disciplinas optativas, os projetos de extensão ofertados no âmbito universitário, propiciam ao aluno experiências além da formação clínica básica. Seguindo os preceitos da proposta curricular, acreditamos que as ofertas de campos de estágios permitem aos graduandos um primeiro contato e talvez, um despertar para futuras áreas de interesse por diferentes especialidades da Odontologia. Neste projeto, especificamente, a proposta permite a amplitude da atuação de acadêmicos propiciando uma vivência no atendimento de um maior número de pacientes, dentro do ambiente hospitalar.

Durante a formação profissional, a articulação entre ensino, pesquisa e extensão, promove o desenvolvimento de competências e habilidades necessárias à construção de um novo perfil do odontólogo, capaz de atuar considerando os problemas e/ou situações de saúde, incluindo as doenças mais prevalentes no perfil epidemiológico nacional, principalmente no caso 
Impacto de um projeto de extensão sobre a formação discente para atuação em ambiente hospitalar

de uma doença crônica como o câncer (COSTA et al., 2013). As atividades de extensão promovem melhoria da qualidade de vida das pessoas que são atendidas, ao passo que contribuem para a Universidade se mantenha apta para corresponder às necessidades externas por meio do conhecimento adquirido com as experiências proporcionadas por essas atividades. Também contribuem para a formação de novos profissionais dotados de consciência social (RODRIGUES et al., 2013).

As experiências vivenciadas no Ambulatório de Odontologia Onco-hematológica, por meio do contato com os pacientes, tanto no ambulatório de odontologia oncológica quanto nas Clínicas Médica I e II do HU/UFSC, viabilizam o despertar do aluno para "perceber o indivíduo como ser integral". A boca e o sistema estomatognático compõem as áreas de atuação do cirurgião-dentista (CFO-63/2005), contudo condutas odontológicas são diretamente influenciadas por patologias sistêmicas diversas (MORAIS et al., 2006). A complexidade dos casos avaliados, torna claro para o aluno extensionista que, toda conduta odontológica deve ser baseada na condição de saúde do paciente, fato este muitas vezes limitador para o atendimento necessário (WALSH, 2010).

Rodrigues et al. (2013) apresentaram o contato dos acadêmicos com o público em geral, onde as teorias aprendidas em sala de aula se concretizam. Os autores mostraram que a extensão universitária traz para a sociedade grandes experiências, tanto na vida dos acadêmicos, quanto na vida das pessoas que usufruem deste aprendizado.

Morais et al. (2006) e Walsh (2010), caracterizaram o programa extensionista como um espaço que intensifica conhecimentos, por meio da diversificação de ambientes e metodologias de aprendizagem, os quais favorecem a efetiva interação entre o ensino, a pesquisa e a extensão. De acordo com os autores, a extensão é capaz de propiciar ao aluno uma formação profissional mais generalista, crítica e sensível, tornando-o apto a entender, preocupar-se e buscar soluções para os anseios da comunidade assistida. Os mesmos avaliaram também a importância de um programa extensionista junto aos alunos e demonstraram que as ações realizadas visam contribuir para o desenvolvimento das aptidões individuais, a capacidade de trabalhar em equipe e a inserção do aluno na comunidade. Desta forma, a extensão universitária é capaz de promover maior convivência, troca de conhecimentos e respeito ao saber alheio.

Outro aspecto positivo configura-se na participação do aluno dentro dos serviços públicos de saúde, propiciando sua aproximação à realidade dos serviços de saúde nacionais, nos quais futuramente o graduando poderá vir a trabalhar. Sendo assim, o projeto busca promover a inter e transdisciplinariedade, inserindo a Odontologia no ambiente hospitalar, promovendo atendimento integral e melhor qualidade de vida aos pacientes (MOIMAZ, 2015). O suporte de 
Impacto de um projeto de extensão sobre a formação discente para atuação em ambiente hospitalar

diferentes profissionais da saúde é importante para agregar conhecimento sobre a evolução da doença e tratamento necessário, visando a recuperação dos pacientes. É nesse ambiente que o aluno da extensão aprende a lidar com pacientes portadores de enfermidades sistêmicas, hospitalizados, que muitas vezes se encontram totalmente dependentes para cuidado e, portanto, se tornam incapazes de manter uma higienização bucal adequada (COSTA et al., 2016). Compreende-se, então, que tratar o paciente oncológico implica em conhecer não apenas a respeito da patologia em si mas, também, saber lidar com sentimentos e com as próprias emoções frente à doença que muitas vezes não tem possibilidade de cura. Nesse meio, o aluno tem a chance de vivenciar as alterações emocionais que a condição de doente impõe ao paciente, a família e aos profissionais da saúde envolvidos (RODRIGUES, 2004).

O bolsista extensionista, juntamente com os professores e residentes do HU, podem acompanhar a recuperação e a melhora na qualidade de vida do paciente. Portanto, o projeto "Ambulatório de Odontologia Oncológica" contribui com o crescimento e maturidade profissional do aluno. Para pacientes, o cuidado ofertado ainda influencia no sucesso do tratamento oncológico, com consequente diminuição da morbi/mortalidade, além de aumentar o acesso aos serviços de odontologia, que atingem não só pacientes, mas também acompanhantes e/ou cuidadores.

Embora os números de produtividade deste serviço não sejam representativos quando comparados aos grandes centros, é indiscutível o ganho atingido. O Trabalho de Conclusão de Curso “Ambulatório Odontológico para Pacientes Onco-hematológicos: Estudo do Impacto Clínico e Econômico" realizado no ano de 2016 buscou, entre outros objetivos, analisar o impacto clínico da implementação do atendimento odontológico do projeto (SALEH, 2016).

De acordo com os resultados obtidos por Saleh (2016) a presença do CD na equipe multiprofissional hospitalar otimizou o diagnóstico de complicações bucais relacionadas à quimioterapia, com uma sensibilidade de diagnósticos de 72,88\%, comparado a 7,27\% sem a presença deste profissional na equipe. Além disso, a presença do CD contribuiu com a melhora na higiene bucal dos pacientes, proporcionando melhor qualidade de vida.

\section{CONSIDERAÇÕES FINAIS}

Do ponto de vista de serviço, os atendimentos odontológicos promovidos pelo projeto de extensão Ambulatório de Odontologia Oncológica - Onco-hematologia no Núcleo de OH e nas Clínicas Médicas I e II do HU/UFSC, são essenciais para a população alvo apresentada nesse estudo e complementam o atendimento multiprofissional ofertado para pacientes diagnosticados com 
Impacto de um projeto de extensão sobre a formação discente para atuação em ambiente hospitalar

doenças onco-hematológicas e em tratamento no HU/UFSC. Do ponto de vista extensionista, o campo de estágio proporciona uma experiência além da aprendida nas clínicas odontológicas da UFSC. O aprendizado do aluno é intensificado em sua formação humanista por promover um contato direto com a finitude humana e seus desenrolares a partir do diagnóstico de uma doença grave como o câncer.

Além do aprendizado técnico científico, a avaliação de risco do indivíduo, medido pela equação necessidade odontológica versus condição sistêmica, exercitado semanalmente em cada atendimento, promove capacitação e autonomia para extensionistas prestes a se formar no curso de graduação em Odontologia.

Esta extensão também propicia o exercício da atuação em equipe multiprofissional, exercitando a interdisciplinaridade, dando uma visão holística do paciente, muito além de suas necessidades odontológicas, desenvolvendo uma visão da amplitude que representa o trabalho do CD no âmbito hospitalar.

A elaboração deste trabalho permitiu estabelecer a necessidade de ampliar este projeto de extensão para um atividade obrigatória dentro do currículo dos cursos de Odontologia, visando uma capacitação a todos os acadêmicos de odontologia, habilitando-os para a atividade em equipes multiprofissional em ambiente hospitalar.

\section{REFERÊNCIAS}

AMANTE, Cláudio José. Projeto Político Pedagógico do Curso de Graduação em Odontologia da Universidade Federal de Santa Catarina. Disponível em: < http://odontologia.ufsc.br/files/2011/07/projeto-pedag\%C3\%B3gico-odonto-UFSC2.pdf>. Acesso em: 16 fev. 2018.

BRASIL. Conselho Federal de Odontologia. Projeto de Lei 2.776/08. Atualizado em 10/04/2013. Disponível em: <http://www.cfo.org.br/destaques/aprovado-projeto-de-lei-quegarante-a-presenca-do-cirurgiao-dentista-nas-utis/>. Acesso em: 22 jun. 2017.

BRASIL. Conselho Federal de Odontologia. Resolução n. 162 de 3 de novembro de 2015. Disponível em: <http://www.crosp.org.br/noticia/ver/2382-odontologia-hospitalarreconhecida-como-habilitao-pelo-cfo.html>. Acesso em: 22 jun. 2017.

BRASIL. Conselho Regional de Odontologia de Santa Catarina. Disponível em: <http://www.crosc.org.br/arquivos_pdf/HABILITACAO.pdf>. Acesso em: 30 nov. 2017a.

BRASIL. Conselho Regional de Odontologia do Piauí. Disponível em: $<$ http://www.cropi.org.br/noticias/a-odontologia-hospitalar-a-serviaco-do-cidadaao-374.html>. Acesso em: 22 jun. 2017b. 
Impacto de um projeto de extensão sobre a formação discente para atuação em ambiente hospitalar

CAMARGO, Elaine C. Odontologia hospitalar é mais do que cirurgia buco-maxilo-facial. Jornal Odonto. Ano VII, n. 98, 2005. Disponível em:

$<$ http://www.jornaldosite.com.br/arquivos/anteriores/elainecamargo/artelainecamargo.98.htm >. Acesso em: 22 jun. 2017.

COSTA, Adriana Cristina Oliva et al. A Odontologia Hospitalar no serviço público do Estado de São Paulo. Revista da Associação Paulista de Cirurgiões-Dentistas. v. 67, p. 224-228, 2013.

COSTA, José Ricardo Souza et al. A Odontologia Hospitalar em Conceitos. Revista da ACBO. v. 25, n.2, p. 211-218, 2016.

JOSHI, Vinod K. Dental treatment planning and management for the mouth cancer patient. Oral Oncol. v. 46, n. 6, p. 475-9, 2010.

MOIMAZ, Suzely Adas Saliba et al. Extensão Universitária como Ferramenta Geradora de Ensino-Aprendizagem e Produtora de Pesquisa. Revista Conexão UEPG, vol. 11, n. 2, p. 140149, ago. 2015.

MORAIS, Tereza Márcia Nascimento de Morais et al. A importância da atuação odontológica em pacientes internados em unidade de terapia intensiva. Revista Brasileira de Terapia Intensiva. v. 18, n. 4, p. 412-7, 2006.

PIVETTA, Hedioneia Maria Foletto Pivetta et al. Ensino, pesquisa e extensão universitária: em busca de uma integração efetiva. Linhas Críticas. v. 16, n. 31, p. 377-390, 2010.

RODRIGUES, Andréia Lilian Lima et al. Contribuições da Extensão Universitária na Sociedade. Cadernos de Graduação - Ciências Humanas e Sociais. v. 1, n.16, p. 141-148, 2013.

RODRIGUES, Inês Gimenes. Cuidados paliativos: análise de conceito. Dissertação (Dissertação em enfermagem) - Escola de Enfermagem de Ribeirão Preto da Universidade de São Paulo. Ribeirão Preto, fl. 231, 2004.

SALEH, Halime Adel. Ambulatório Odontológico para Pacientes Onco-hematológicos: Estudo do Impacto Clínico e Econômico. Trabalho de Conclusão de Curso (Trabalho de Conclusão de Curso em Odontologia) - Universidade Federal de Santa Catarina. fl. 86, 2016.

SÃO PAULO (Estado). Secretaria de Saúde. Manual de odontologia hospitalar. São Paulo, 2012. Disponível em: <http://www.saude.sp.gov.br/resources/ses/perfil/profissional-dasaude/grupo-tecnico-de-acoes-estrategicas-gtae/manual-de-odontologia-

hospitalar/manual_odonto.pdf>. Acessado em: 25 ago. 2017.

SANTOS, Paulo Silva et al. A equipe multidisciplinar e a ação do cirurgião-dentista nos pacientes transplantados renais: uma revisão integrativa. Revista brasileira de medicina. v.73, n. 2, p. 2632, 2016.

SONIS, Stephen T; FAZIO, Robert C; FANG, Leslie ST. Princípios e prática de medicina oral. Rio de Janeiro: Guanabara Koogan, p. 225-235, 1996.

UFSC. Currículo do Curso de Graduação em Odontologia. Disponível em: <http://cagr.ufsc.br/relatorios/curriculoCurso?=104>. Acesso em: 21 nov. 2017. 
Impacto de um projeto de extensão sobre a formação discente para atuação em ambiente hospitalar

UFSC. Plano de Desenvolvimento Institucional. Disponível em: <http://pdi.ufsc.br/files/2015/05/PDI-2015-2019-1.pdf>. Acesso em: 21 nov. 2017.

WALSH, Laurence J. Clinical assessment and management of the oral environment in the oncology patient. Aust Dent J. v. 55, Suppl. 1, p. 66-77, 2010.

Recebido em: 28/06/2018

Aceito em: $11 / 12 / 2018$ 